\title{
NIVELES DE NARCISISMO EN EDUCADORES Y SUS EFECTOS EN LA FUNCIÓN TUTORIAL
}

Orlando Cerna Dorregaray

\section{RESUMEN}

La presente investigación desea conocer la relación que existe entre la estructura de personalidad narcisista de los educadores con el desempeño de la función tutorial de los mismos. Es un estudio descriptivo correlacional, donde se describen los niveles de narcisismo en los educadores y se los relaciona con la percepción que los educandos tienen de la acción tutorial que realizan sus profesores tutores. Se evaluó a 172 profesores de los cuales 84 son tutores y a 3765 estudiantes de secundaria de nueve colegios religiosos del Perú. Un aspecto a destacar del presente estudio es que hay mucha investigación de la tutoría en función del estudiante, pero no así desde la perspectiva de la personalidad del educador, como si la misma fuera negada y no se vinculara con el trabajo educativo. Lo que se buscó en el estudio es demostrar la importancia que tiene la variable personalidad del educador en las acciones tutoriales que los educadores realizan; en especial los niveles de narcisismo de los educadores y cómo estos están relacionados con la percepción de los estudiantes de la acción tutorial individual, grupal y con los padres de familia.

Los aspectos teóricos que sustentan las preguntas del estudio son propuestas desde la teoría psicoanalítica en general y desde la teoría de las relaciones de objeto de Melanie Klein y desde los estudios contemporáneos de Otto Kernberg, donde el objeto es definido como aquello con lo que el sujeto interactúa y que puede ser de diverso tipo. Esta perspectiva ayuda a comprender el fenómeno narcisista en la personalidad de los educadores. Para medir el narcisismo se utilizó la Escala N15 de Trechera desarrollada en España y adaptada para el presente estudio. Por el lado de la variable de la acción tutorial se parte de una concepción humanística de la psicología postulada por Rogers, donde el centro de la educación es la persona del educando. Para evaluar la acción tutorial se utilizó la Encuesta de Evaluación de la Acción Tutorial creada por Galve y García en España y adaptada para esta investigación. La encuesta evalúa tres áreas de la acción tutorial: la Relación Estudiante-Tutor, Grupo-tutor y Padres-Tutor; también brinda un resultado de la acción tutorial general. 
Los análisis de confiabilidad y validez de los instrumentos en la población estudiada fueron adecuados. En la Escala N15 que mide narcisismo se alcanzó una confiabilidad Alfa de Cronbach para el grupo de tutores de 0,74 y para la muestra total de educadores de 0,78 . También se realizaron análisis de validez de contenido utilizando el criterio de jueces, donde todos los ítemes a excepción de uno solo pasaron el criterio mínimo de 0,60 utilizando la fórmula de Aiken. En lo que respecta a la Encuesta de Acción Tutorial alcanzó una confiabilidad de Alfa de Cronbach de 0.79.

Con los resultados anteriores se establecieron los niveles de narcisismo de los educadores y los niveles de acción tutorial evaluados por la percepción de los estudiantes. Posteriormente se realizaron los análisis estadísticos que integraron las características de la personalidad narcisista de los educadores y las características de la acción tutorial medida a través de la percepción de los educandos.

Dentro de los hallazgos destaca la relación que existe entre el narcisismo de los educadores y la acción tutorial evaluada por los estudiantes. También se encontró que el $73.5 \%$ de los tutores se ubican en un nivel medio de narcisismo, lo que indica que no estamos en un nivel patológico de narcisismo. De todos modos estos niveles medios influyen en la acción tutorial que es percibida por los estudiantes como no totalmente favorable o beneficiosa para ellos. Por otro lado, un dato interesante es percibir por parte de los educandos que los niveles altos son favorables para la acción tutorial. Esto estaría relacionado con la necesidad que tiene el adolescente de identificarse con personas a las que llegan a considerar muchas veces "ídolos". También se encuentra que se percibe como desfavorable para la acción tutorial los niveles bajos o muy bajos de narcisismo. Esto puede ser interpretado, como la necesidad de los adolescentes de tener tutores con un nivel de autoamor normal, cercano a la autoestima e incluso que este autoamor sea alto.

Otro dato que se recoge del estudio es que la percepción de la Acción Tutorial es más favorable en los colegios de provincias en comparación con los colegios de Lima. Son los estudiantes de los primeros años de secundaria los que perciben la acción tutorial como más favorable y va disminuyendo conforme avanzan en el grado de estudio; lo cual demuestra otro aspecto teórico de desarrollo adolescente: que conforme avanza en su edad, va sintiendo la necesidad de alejarse más, tanto de las figuras parentales como de las figuras de autoridad, como en este caso son los tutores, todo con el objetivo de lograr su propia identidad.

Otro hallazgo que se diferencia del resto es constatar que los niveles altos de narcisismo son perjudiciales para la relación grupo-tutor. Es decir que las acciones de liderazgo-narcisismo no contribuyen a la orientación grupal, a revisar los temas de interés de los estudiantes, a mejorar las relaciones interpersonales del grupo consigo mismo y con el tutor y con otros 
profesores. De tal forma que un narcisismo en niveles altos o malignos como los llama Kernberg, es de una influencia negativa para la orientación tutorial a nivel grupal.

De este modo, la presente investigación, responde a una preocupación importante de la educación en nuestro país, donde la tutoría se constituye en el eje central que permite cumplir los objetivos de la formación integral de los adolescentes. Permite abrir el camino para señalar la importancia de la personalidad del educador en la formación de los estudiantes, formación de su mente, de su afectividad y de sus habilidades para relacionarse con los otros. Nos lleva a reconocer que la tutoría en particular y la educación en general requieren de una psicología que sea clínica y educativa, donde el enfoque psicoanalítico sea considerado en la medida que involucra la personalidad del educador. Es un estudio que nos confronta con nuestro propio narcisismo y que nos invita a educar desde el vínculo educador-educando, relación y vínculo que nos permite ser más y mejores seres humanos.

\section{PROBLEMA}

La educación tiene entre sus principales fines la formación integral del ser humano, es decir, tanto los aspectos cognitivos como afectivos. Esta formación requiere la participación de varios factores, entre ellos la formación profesional del docente en los aspectos teóricos y metodológicos que le permitan lograr los objetivos educacionales. Dentro de su formación, sin embargo, el perfil humano del educador como persona muchas veces se deja de lado, como si su personalidad, y su subjetividad no influyeran en su labor docente, en sus funciones académicas y sobre todo en las tutoriales. Es en estas funciones de tutoría donde los profesores tutores aparte de tener que recibir una formación especializada, deben de tener conocimiento de su estructura de personalidad y cómo esta facilita u obstaculiza el logro de sus funciones como tutor.

En el trabajo psicopedagógico de coordinación y asesoramiento con tutores a nivel de secundaria, se ha podido observar y evaluar empíricamente que uno de los principales obstáculos en el logro de los objetivos de la tutoría radica en la presencia de ciertas características de personalidad narcisista en algunos educadores. Se aprecia que una personalidad narcisista tiene una imagen distorsionada de sí mismo, falta de empatía, hipersensibilidad a la evaluación de los demás y dificultades en las relaciones interpersonales. Esto condujo a plantear una investigación que permita examinar si la personalidad de los educadores tutores tiene implicancia en sus funciones como tutor. Así el estudio se propuso responder la siguiente interrogante: ¿En qué medida se relaciona la estructura de personalidad narcisista de los educadores con el desempeño de la función tutorial de los mismos?

En nuestro medio los estudios sobre personalidad narcisista se han realizado a partir de la 
psicología clínica y especialmente desde la psicoterapia. Los especialistas señalan que la estructura de personalidad narcisista es la psicopatología del fin del siglo XX e inicios del siglo XXI, tal como lo era la histeria a fines de siglo XIX. Incluso se postula que estamos viviendo una cultura narcisista, que privilegia el culto al cuerpo, al excesivo cuidado y afecto hacia uno mismo, que va más alla de un narcisismo normal que se asemeja a la autoestima y que se acerca más hacia un narcisismo maligno (Kernberg, 1998) que puede conducir al individuo hacia su autodestrucción.

Los estudios que se relacionan con esta investigación en el Perú es la realizada por Gheiler (2001) con sus aportes sobre la aplicación del psicoanálisis a la educación, quien postula la necesidad de buscar el desarrollo personal y psicológico del educador como requisito para ejercer la función de tutores. En Latinoamérica se han realizado avances significativos tanto en Argentina como en Brasil, postulando y enfocando la psicopedagogía desde una visión psicoanalítica. Precisamente nuestro acercamiento al tema se realiza desde los trabajos presentados por Cukier (1997) quien acuña el término didactogenia (patología inducida por la enseñanza) para explicar que es un educador con características narcisistas quien provoca en los educandos miedo, sometimiento, confusión y depresión. Si esto ocurre en la enseñanza y aprendizaje en general qué puede estar ocurriendo a nivel de las funciones de la tutoría que se concretizan en labores de consejería (tutoría individual) y de liderazgo (tutoría grupal). Esta investigación estudió la influencia de la variable personalidad narcisista en las funciones de tutoría antes descritas y su repercusión en el quehacer educativo en general.

Nuestra posición teórica se ubica en el enfoque psicoanalítico en general y en particular en la propuesta de Klein y de sus seguidores como Kernberg (1998) quienes proponen que toda la vida psíquica humana se funda en las relaciones con otros desde el nacimiento. Nos referimos a relaciones de cuidado y atenciones. Muchas veces, en la medida que la realidad frustra las relaciones de afecto, el proceso de identificación con el self, hace que el individuo para defenderse de esta carencia o exceso de afecto del medio, se fije en sí mismo, es decir, el afecto se dirige hacia uno mismo, produciéndose una identificación narcisista que en algunos puede ser normal y en otros patológica. Desde esta perspectiva, todos tenemos un nivel de narcisismo. Nos preguntamos si el narcisismo de los educadores es normal o patológico y en qué medida estos niveles influyen en la labor tutorial que todo educador debe realizar.

Para la evaluación del narcisismo se utiliza la Prueba de Narcisismo de Trechera (1994) que fue necesario validar para este estudio ya que fue un instrumento creado en España, que se basa en el NPI (Inventario de Personalidad Narcisista) de Raskin y Hall (1988). Este instrumento se encuentra dentro del contexto psicoanalítico y permitió diagnosticar la personalidad narcisista de los educadores. Por otro lado, para la evaluación de la acción 
tutorial se utilizó la Escala de Evaluación de la Acción Tutorial de Galve y García (1997) que también se validó para la muestra en estudio; con ella los estudiantes evalúan la acción tutorial en base a las acciones individuales y grupales que todo tutor realiza dentro de su labor.

En conclusión y en base a lo expuesto surgió la siguiente interrogante: ¿En qué medida se relaciona la estructura de personalidad narcisista de los educadores con el desempeño de la función tutorial? Interrogante que fue contestada desde dos perspectivas: el enfoque teórico psicoanalítico para explicar la personalidad narcisista y la pedagogía humanística para entender la función tutorial dentro de una educación que busca el desarrollo integral del educando. Ambos enfoques y su metodología permitieron un acercamiento objetivo y científico del presente estudio.

\section{MÉTODO}

\section{Tipo de investigación}

Se realiza una investigación descriptiva correlacional en la medida que nos interesa conocer las características de la personalidad de los educadores y relacionarlas con las características de la función tutorial evaluada por los educandos.

\section{PARTICIPANTES:}

\section{Muestra}

Conformada por dos grupos. Uno de profesores y otro de estudiantes. El grupo de profesores son del nivel de secundaria de colegios religiosos del Perú que constituyen un total de 172. De los cuales, para el estudio se escogieron 83 docentes que ejercen la función de tutores. Luego de las depuraciones del caso, se trabajó con 81 tutores en la medida que dos de ellos no proporcionaron algunos datos de la encuesta. Existen más tutores varones (58) que mujeres (25). Existen más tutores de Letras (55) que de Ciencias (28). La edad promedio del tutor es 39,7 años. Fueron evaluados con la escala N15 todos los tutores de secundaria.

El grupo de estudiantes, la población escolar evaluada llega a un total de 3765 educandos. Fueron evaluados todos con la Escala de Acción Tutorial. Se dividió a los estudiantes en dos grupos, los colegios de provincia y los colegios de Lima.

La muestra es intencional no probabilística. 


\section{Técnicas de recolección de datos}

Para medir la estructura de personalidad narcisista se utilizó la Prueba de Narcisismo de Trechera conocida como N15, la cual tiene una confiabilidad Alfa de Cronbach de 0.83 y consta de 28 ítemes. La prueba mide tres factores y dos subescalas. Los factores son: Narcisismo, maquiavelismo y dominancia. Las subescalas son: Falta de empatía y exhibicionismo.

Para la evaluación de la acción tutorial se utilizó la Escala de Acción Tutorial de Galve y García, que mide la función tutorial a nivel individual y a nivel grupal y que fue adaptada para la presente investigación.

Luego de los análisis estadísticos pertinentes se decidió trabajar con la Escala N15 en su versión total. En la tabla 1 se muestran las estadísticas descriptivas de la Escala N15 así como los niveles de narcisismo obtenidos para los docentes evaluados usando la distribución normal de N15. De este modo tenemos un baremo que nos permite el diagnóstico de Narcisismo en educadores de la muestra de estudio y que puede ser utilizado para muestras similares.

TABLA 1:

CATEGORÍAS DIAGNÓSTICAS DEL NARCISISMO PARA LA MUESTRA DE COLEGIOS RELIGIOSOS SEGÚN EL PUNTAJE OBTENIDO EN LA ESCALA N15

\begin{tabular}{c|c} 
PUNTUACIONES & NIVELES DE NARCISISMO \\
\hline 68 o más & Muy alto \\
\hline $59-67$ & Alto \\
\hline $41-58$ & Medio \\
\hline $31-40$ & Bajo \\
\hline 30 o menos & Muy bajo \\
\hline Media & 48.9 \\
\hline D.E & 8.9 \\
\hline
\end{tabular}

En lo que respecta a la función tutorial, luego de los análisis estadísticos de la Escala de Acción Tutorial se decidió trabajar con el cuestionario completo y sus áreas. En la tabla 2 se presentan las estadísticas descriptivas del cuestionario y de sus áreas así como los niveles de la Acción tutorial obtenidos usando percentiles ya que no se tiene distribución 
normal. Se presentan baremos que permiten un diagnóstico de la acción tutorial desde la percepción de los estudiantes y que será útil para la muestra de estudio y para similares a ella.

TABLA 2

BAREMOS DE LA ACCIÓN TUTORIAL PARA LA MUESTRA DE DOCENTES DE COLEGIOS RELIGIOSOS

\begin{tabular}{c|c|c|c|c|c}
\multirow{2}{*}{ NIVELES } & PERCENTILES & $\begin{array}{c}\text { ALUMNO- } \\
\text { TUTOR }\end{array}$ & $\begin{array}{c}\text { GRUPO- } \\
\text { TUTOR }\end{array}$ & $\begin{array}{c}\text { PADRES- } \\
\text { TUTOR }\end{array}$ & $\begin{array}{c}\text { ACCIÓN } \\
\text { TUTORIAL } \\
\text { TOTAL }\end{array}$ \\
\hline \multirow{2}{*}{ ALT0 } & P70 o más & $18-20$ & 10 & $3-16$ & $39-46$ \\
\hline \multirow{2}{*}{ BAJ0 } & P40 - P69 & $14-17$ & $8-9$ & $11-12$ & $33-38$ \\
\hline \multirow{2}{*}{} & P39 o menos & $0-30$ & $0-7$ & $0-10$ & $0-32$ \\
\cline { 2 - 6 } & Media & 14.8 & 8.1 & 11.2 & 3765 \\
\cline { 2 - 6 } & D.E & 4.0 & 2.2 & 3.3 & 7.3 \\
\cline { 2 - 6 } & Mínimo & 1 & 0 & 1 & 3 \\
\cline { 2 - 6 } & Máximo & 20 & 10 & 16 & 46 \\
\hline
\end{tabular}

\section{Diseño de investigación}

El diseño de investigación es el descriptivo comparativo correlacional. Se describen y comparan las evaluaciones de la función tutorial por parte de los educandos de acuerdo a los niveles de narcisismo de los tutores. Luego se establecen correlaciones entre dichas variables para determinar si existe algún grado de asociación entre las mismas. Es decir, estamos ante un diseño de diferencias de grupo. Se compararon las apreciaciones (evaluaciones de la función tutorial), según el nivel de personalidad narcisista encontrado en los educadores.

\section{Resultados}

El objetivo del presente estudio fue analizar la influencia de los niveles de narcisismo de los educadores en la acción tutorial que los mismos realizan. Se presentan los resultados siguiendo el siguiente orden: 
1. Resultados de la acción tutorial en el ámbito de los estudiantes.

2. Resultados del narcisismo en el ámbito de los tutores

3. Análisis para explicar la acción tutorial en función de las características de personalidad de los docentes.

\section{Resultados de la acción tutorial en el ámbito del estudiante}

Puede observarse que, por procedencia, los estudiantes de provincias obtienen valores medios superiores en las áreas: estudiante-tutor, grupo-tutor y padres-tutor.

Por otro lado, son los estudiantes de primero de secundaria los que obtienen mayor valor medio en las puntuaciones estudiante- tutor en relación a los de Lima. Los estudiantes de quinto son los que obtienen el menor valor medio en esta área, en relación a los demás grados de estudio.

En el área de relación grupo-tutor, son los estudiantes de segundo los que obtienen valor mayor medio que los demás grados y los estudiantes de cuarto y quinto obtienen el menor valor medio.

En el área padres-tutor, son los estudiantes de primer grado los que obtienen mayor valor medio y los estudiantes de quinto los que obtienen menor valor medio. Todo lo anterior lo podemos apreciar en la tabla 3.

TABLA 3

VALORES MEDIOS Y DESVIACIÓN ESTÁNDAR PARA LA ACCIÓN TUTORIAL CONSIDERANDO LA PROCEDENCIA Y GRADO DE ESTUDIO

\begin{tabular}{|c|c|c|c|c|c|c|c|}
\hline \multirow[t]{2}{*}{ ÁREA } & \multicolumn{2}{|c|}{$\begin{array}{l}\text { PROCEDENCIA } \\
\text { DEL COLEGIO }\end{array}$} & \multicolumn{5}{|c|}{ GRADO DEL ESTUDIANTE } \\
\hline & PROVINCIA & LIMA & PRIMERO & SEGUNDO & TERCERO & CUARTO & QUINTO \\
\hline $\begin{array}{c}\text { ESTUDIANTE- } \\
\text { TUTOR }\end{array}$ & $\begin{array}{l}\text { M } 15.6 \\
\text { DE } 3.7\end{array}$ & $\begin{array}{c}14.2 \\
4.1\end{array}$ & 15.7 & 15.0 & 14.8 & 14.2 & 14.1 \\
\hline GRUPO-TUTOR & $\begin{array}{ll}\text { M } & 8.7 \\
\text { DE } & 1,9\end{array}$ & $\begin{array}{l}7.7 \\
2,3\end{array}$ & 8.3 & 8,4 & 8.1 & 7.9 & 7.9 \\
\hline PADRES-TUTOR & $\begin{array}{cc}M & 12.3 \\
D E & 3.0\end{array}$ & $\begin{array}{l}10.4 \\
3.3\end{array}$ & 11.6 & 11.4 & 11.1 & 11.0 & 10.9 \\
\hline
\end{tabular}




\section{Resultados del narcisismo en el ámbito de los tutores}

Los resultados nos muestran a 61 tutores con un nivel de autoamor de nivel medio; Es decir que los niveles de narcisismo, en la mayoría de tutores, no llegan a un nivel patológico. Solo hay 7 tutores que se ubican en los niveles altos o muy altos. Estos deberían recibir orientación psicológica en la medida que pueden influir en la función tutorial. Por otro lado, se observa un grupo de tutores que se ubica en los niveles bajos o muy bajos de narcisismo. Esto implica que estos tutores no se encuentran centrados en su propio yo o persona y que sus niveles de autoamor son bajos. Todo esto lo podemos apreciar en la tabla 4.

TABLA 4

NIVEL DE NARCISISMO EN LA MUESTRA DE TUTORES DE LOS COLEGIOS RELIGIOSOS

\begin{tabular}{c|c|c}
\hline NIVELES DE NARCISISMO & FRECUENCIA & PORCENTAJE \\
\hline MUY BAJO & 1 & 1.2 \\
\hline BAJO & 14 & 16.9 \\
\hline MEDIO & 61 & 73.5 \\
\hline ALTO & 6 & 7.2 \\
\hline MUY ALTO & 1 & 1.2 \\
\hline TOTAL & 83 & 100 \\
\hline
\end{tabular}

3. Análisis para explicar la acción tutorial en función de las características de personalidad de los docentes

Las comparaciones entre las áreas de la acción tutorial de los estudiantes, de acuerdo a los niveles de narcisismo de los tutores, encuentra diferencias muy significativas en todas las áreas evaluadas en la encuesta y en la percepción de la acción tutorial de los estudiantes.

Se observa que hay una mayor proporción de estudiantes con percepción de la acción tutorial por debajo de la mediana entre los que tienen tutores con niveles de narcisismo muy bajo, bajo y medio. Esta situación va cambiando conforme el estudiante pertenece a tutores que tiene niveles más altos de narcisismo, salvo en la percepción grupo tutor de los estudiantes con tutores con alto narcisismo.

Hay una mayor proporción de estudiantes con percepción de la acción tutorial por encima de la mediana entre los que tienen tutores con niveles de narcisismo muy alto. 
En conclusión, estos resultados nos indican que existen diferencias significativas entre la percepción de la acción tutorial en función de los niveles de narcisismo de los educadores, con lo cual se comprueba la hipótesis de que la variable personalidad de los tutores sí esta relacionada con la acción tutorial que estos realizan.

En este sentido se aprecia que, a menores niveles de narcisismo, la percepción de la acción tutorial es percibida por el educando como menos beneficiosa o útil en su educación. Esto contrasta con los niveles altos o muy altos de narcisismo, en donde los estudiantes perciben que la acción tutorial es beneficiosa o útil para ellos.

Lo anterior contradice las hipótesis empíricas que señalan que este tipo de personalidad puede ser perjudicial. En todo caso, los adolescentes pueden estar percibiendo este narcisismo alto, no como una patología o un trastorno de personalidad, sino como un nivel alto de autoestima del profesor. Un dato importante es cuando nos fijamos en el área grupo-tutor. Se aprecia que los niveles altos de narcisismo no son percibidos como beneficiosos o útiles para la acción tutorial grupal. Es decir, un líder narcisista resulta perjudicial para los grupos que tiene a cargo. Todo esto lo podemos percibir en la tabla 5.

TABLA 5

PRUEBA DE COMPARACIÓN DE MEDIANAS PARA LA ACCIÓN TUTORIAL CONSIDERANDO LOS NIVELES DE NARCISISMO EN LOS TUTORES

\begin{tabular}{|c|c|c|c|c|c|}
\hline $\begin{array}{l}\text { NIVELES DE } \\
\text { NARCISISMO } \\
\text { EN EL TUTOR }\end{array}$ & $\begin{array}{c}\text { MEDIANA } \\
\quad> \\
<\end{array}$ & $\begin{array}{c}\text { ESTUDIANTE } \\
\text {-TUTOR }\end{array}$ & $\begin{array}{l}\text { GRUPO - } \\
\text { TUTOR }\end{array}$ & $\begin{array}{c}\text { PADRES } \\
\text { TUTOR }\end{array}$ & $\begin{array}{c}\text { ACCIÓN } \\
\text { TUTORIAL }\end{array}$ \\
\hline MUY BAJO & & $\begin{array}{l}36.8 \\
63.2 \\
\end{array}$ & $\begin{array}{l}21.1 \\
68-9\end{array}$ & $\begin{array}{l}26.3 \\
73.7\end{array}$ & $\begin{array}{l}42.1 \\
57-9\end{array}$ \\
\hline BAJO & & $\begin{array}{l}34.4 \\
65.6\end{array}$ & $\begin{array}{l}29.5 \\
70.5\end{array}$ & $\begin{array}{l}31.8 \\
68.2\end{array}$ & $\begin{array}{l}36.6 \\
63.4\end{array}$ \\
\hline MEDIO & & $\begin{array}{l}42.8 \\
57.2\end{array}$ & $\begin{array}{l}41.6 \\
58.4\end{array}$ & $\begin{array}{l}40.6 \\
59.4\end{array}$ & $\begin{array}{l}50.0 \\
50.0\end{array}$ \\
\hline ALTO & & $\begin{array}{l}49.5 \\
50.5\end{array}$ & $\begin{array}{l}37.7 \\
62.3\end{array}$ & $\begin{array}{l}50.0 \\
50.0\end{array}$ & $\begin{array}{l}61.4 \\
38.6\end{array}$ \\
\hline MUY ALTO & & $\begin{array}{l}61.7 \\
38.3\end{array}$ & $\begin{array}{l}57.4 \\
42.6\end{array}$ & $\begin{array}{l}70.2 \\
29.8\end{array}$ & $\begin{array}{l}76.6 \\
24.4\end{array}$ \\
\hline & Sig. & $0.000^{\star *}$ & $0.000^{\star *}$ & $0.000^{\star *}$ & $0.000^{\star *}$ \\
\hline
\end{tabular}




\section{Discusión}

Uno de los hallazgos importantes del estudio ha sido que la percepción del estudiante de provincia es más de cercanía con el tutor y más favorable para su educación en general en comparación con el de la capital. En la provincia todavía se resisten a los cambios de la postmodernidad; el contacto personal es más real, lo cual nos aleja del individualismo que se propugna en las sociedades postmodernas como señala Lipovetsky (1991) citado por Trechera (1996).

Otro dato importante es la percepción de los estudiantes de la acción tutorial que varía de acuerdo al grado de estudio: los de $1^{0}$ y $2^{0}$ de secundaria perciben la relación alumno tutor como más cercana que los estudiantes de $4^{0}$ y $5^{0}$ de secundaria. Puede entenderse desde la postura de Erikson $(1974,1983)$ donde la búsqueda de identidad primero pasa por una dependencia a otras figuras diferentes a la de los padres, en este caso los maestros, que se inicia con admiración en los primeros años de secundaria y termina con cuestionamientos al final de la secundaria cuando se logra la propia identidad.

Lo mismo puede ocurrir con la relación padres-tutor, donde los adolescentes puberales ,según Carbajal(1994), no vivencian la crisis de identidad en el mismo sentido que los adolescentes nucleares que se encuentran en $4^{0}$ y $5^{0}$ de secundaria. Por ello los puberales desean que la reunión con sus padres se dé y la perciben positiva.

Otro hallazgo nuclear se refiere a los educadores que presentan niveles medios de narcisismo: son evaluados desfavorablemente en su acción tutorial individual con sus estudiantes, lo cual indica que basta este nivel de narcisismo para que se dificulte la relación personal con el estudiante. Esto confirma nuestra hipótesis de la influencia negativa de una personalidad narcisista de los educadores. Tal como lo sostienen teóricamente Cukier (1997) y Manoni (1997) al plantear que el educador narcisista está más preocupado en satisfacer sus deseos personales que los de sus estudiantes, los cuales pueden ser anulados con ciertas estrategias mal llamadas pedagógicas.

No debemos olvidar que también se percibe como más favorable por los adolescentes los tutores con niveles altos de narcisismo, esto cuando se evalúa al tutor en su función de orientador individual, es decir a mayor narcisismo mejor percepción como tutor. Podemos explicar este resultado teniendo en cuenta lo que señala Kernberg (1999) quien sostiene que los adolescentes tienen la necesidad de una idealización exagerada de los que ellos consideran sus ídolos. Estos adolescentes pueden estar gratificando sus necesidades narcisistas a través de la relación personal que establecen con ellos. Esta identificación estaría explicando los resultados de la necesidad de un tutor más narcisista en la relación o el vínculo que se establece entre el estudiante y el tutor. En todo caso, como señala Kernberg, esta identificación es normal y estaría hablando de un grupo de 
adolescentes que pueden ser en algunos casos normales y en otros casos neuróticos, mas no con características de personalidades patológicas del tipo borderline o narcisista en la medida que en lugar de una identificación que se expresa en nuestro estudio a través de una relación favorable en la relación estudiante-tutor, se expresaría en conflictos de desadaptación severa.

Finalmente, discutiremos el hallazgo del estudio que hace referencia a los niveles altos de narcisismo en los educadores: los estudiantes perciben desfavorablemente la acción tutorial grupo-tutor. Esto implica que el liderazgo ejercido por un tutor narcisista es perjudicial para que los adolescentes se sientan entendidos en sus intereses grupales, tales como la solución de problemas grupales y la mejora de las relaciones interpersonales con el resto de docentes que ingresan al aula a enseñarles. Esto se explica según Kernberg en la medida que los educadores narcisistas presentan mecanismos de proyección exagerados y ampliados debido a la concentración de poder en el líder, haciendo que la profecía autocumplida del educador narcisista dificulte las relaciones interpersonales del mismo y se deteriore la relación con el grupo por las acciones del tutor. Otra característica importante según Kernberg en estos líderes narcisistas y en nuestro caso educadores narcisistas, se refiere a la incapacidad para escuchar puntos de vista diferentes a los suyos y las defensas que realiza frente a su envidia inconsciente, que hacen que las relaciones con los educandos y demás colegas entre en continuos conflictos.

Por lo tanto, los resultados confirman que es necesario considerar la influencia de la variable personalidad de los educadores en la labor tutorial que tienen que ejercer, con el objetivo de formar integralmente a sus educandos.

Así, la tutoría que se propone debe partir del mundo interno, intrapsíquico, del tutor y del educando. Una tutoría que considere la personalidad del tutor, el narcisismo de los educadores y educandos. Solo a partir del reconocimiento de que necesitamos ser primero dueños de nosotros mismos, es decir dueños de nuestra psicología como adultos, podremos entendernos y ayudar a que los que están en desarrollo logren también entenderse y ser dueños de sí mismos.

No podemos educar negando la vida emocional, los niveles de narcisismo y vivir y educar alejados del mundo interno de los estudiantes y de las expectativas y proyecciones que ellos colocan en los educadores. Seamos capaces de dejar de repetir compulsivamente lo que ya antes hicimos y no fue suficiente. Elijamos por lo tanto un camino distinto y difícil al educar, difícil porque nos cuestiona y nos crea conflictos, pero que nos garantiza que al final seremos seres humanos más integrados y comprometidos con los demás. 


\section{REFERENCIAS BIBLIOGRÁFICAS}

Caplansky, M. (1998). Narcisismo. Conferencia en el curso introductorio de Formación en Psicoterapia Psicoanalítica. CPPL Lima.

Carbajal, G. (1993). La aventura de la metamorfosis. Colombia: Tiresias

Cerna, 0. (2003). Niveles de narcisismo en educadores y sus efectos en la función tutorial. Tesis para optar al grado de Doctor en Educación. Lima: Universidad Marcelino Champagnat.

Cukier, J. (1997). Psicopedagogía Analítica. Buenos Aires: Inédito.

Erikson, E. (1974). Identidad, juventud y crisis. Buenos Aires: Paidós.

Galve, J.; García, E. (1992). La acción tutorial en la enseñanza no universitaria. Madrid.

García, J.M: (1995). Narcisismo y efectividad docente en profesores universitarios. Tesis presentada en la Universidad Iberoamericana. México DF: Universidad Iberoamericana

Gheiler, M. (2003). Psicoanálisis, Educación y Responsabilidad Social. Lima: Centro de Desarrollo Humano y Creatividad; Centro de Investigación de la Universidad del Pacifico. Universidad Nacional Mayor de San Marcos.

Kernberg, O. (1999). Ideología, conflicto y liderazgo en grupos y organizaciones. Barcelona: Paidós.

Klein, M. (1994). Obras completas. Barcelona: Paidós

Trahtemberg. L. (2003). Educación, salud mental, derechos humanos y responsabilidad social: estamos mal, no queremos mejorar, pero iqué para estar bien? Psicoanálisis, Educación y Responsabilidad Social. Lima: Centro de Desarrollo Humano y Creatividad; Centro de Investigación de la Universidad del Pacifico. Universidad Nacional Mayor de San Marcos.

Trechera, J.L. (1996). ¿Qué es el narcisismo? Bilbao: Desclée de Brouwer. 